\title{
Estudo sobre a vida útil residual de duas passarelas de pedestres localizadas na
}

\section{rodovia BR-101/PE}

\author{
Study on the residual service life of two pedestrian walkways located in BR-101 / PE \\ Estudio sobre la vida útil residual de dos puentes peatonales situados en la BR-101/PE
}

Recebido: 19/09/2021 | Revisado: 25/09/2021 | Aceito: 28/09/2021 | Publicado: 01/10/2021

\author{
Clayton José Gomes Silva \\ ORCID: https://orcid.org/0000-0003-0072-2807 \\ Universidade Federal de Pernambuco, Brasil \\ E-mail: claytonjgsilva@gmail.com \\ José Afonso Pereira Vitório \\ ORCID: https://orcid.org/0000-0003-3981-3300 \\ Universidade de Pernambuco, Brasil \\ E-mail: afonsovitorio@gmail.com \\ Maria Helena Melo Ferrer de Morais \\ ORCID: https://orcid.org/0000-0003-3326-2042 \\ Departamento Nacional de Infraestrutura de Transportes, Brasil \\ E-mail: helenaferrer@hotmail.com \\ Arnaldo Manoel Pereira Carneiro \\ ORCID: https://orcid.org/0000-0002-4279-7156 \\ Universidade Federal de Pernambuco, Brasil \\ E-mail: arnaldo.carneiro@ufpe.br \\ Romilde Almeida de Oliveira \\ ORCID: https://orcid.org/0000-0002-6786-9080 \\ Universidade Federal de Pernambuco, Brasil \\ E-mail: romildealmeida@gmail.com
}

\begin{abstract}
Resumo
O modal rodoviário se destaca como o sistema mais utilizado no Brasil para o transporte de cargas e de pessoas. Dessa forma, é extremamente importante assegurar recursos que visem à manutenção das rodovias e de suas Obras de Arte Especiais - OAEs (pontes, viadutos e passarelas de pedestres). Estas últimas, que constituem o objeto deste estudo, desempenham um papel indispensável para a segurança e o conforto dos usuários que realizam a travessia das rodovias. Entretanto, é possível verificar que considerável quantidade dessas estruturas se encontra em péssimas condições de conservação, seja pela ação da deterioração que ocorre ao longo do tempo, por deficiências estruturais provenientes do choque de veículos, por vandalismo, ou outros fatores que afetam a sua vida útil. Nesse sentido, este trabalho tem como objetivo estimar a vida útil de duas passarelas de concreto situadas na rodovia federal BR-101/PE, por meio da aplicação do conceito de vida útil estabelecido pelas normas ISO 15686 (2011) e NBR 15575-1 (ABNT, 2021) e NBR 15575-2 (ABNT, 2013). Para tanto, foram utilizados os dados obtidos pelo Sistema de Gerenciamento de Obras de Arte Especiais (SGO) do DNIT. As informações das anomalias e das respectivas condições de estabilidade estrutural possibilitaram a utilização do Método dos Fatores para a obtenção da vida útil estimada e residual das obras estudadas. Os resultados obtidos mostraram que a metodologia pode ser empregada em estudos de casos nos quais não existam um histórico de inspeções regulares de OAEs.
\end{abstract}

Palavras-chave: Passarelas de pedestres; Rodovia; Deterioração; Inspeção; Vida útil.

\begin{abstract}
The road modal stands out as the most used system in Brazil for cargo transportation and people. Thus, it is extremely important to ensure resources for the maintenance of highways and their Special Engineering Structures - SES (bridges, viaducts and pedestrian walkways). The latter, which are the object of this study, play an indispensable role for the safety and comfort of users who cross the highways. However, it is possible to verify that a considerable number of these structures are in very poor conservation conditions, either due to the deterioration that occurs over time, structural deficiencies resulting from vehicle collisions, vandalism, or other factors that affect their useful life. In this sense, this paper aims to estimate the service life of two concrete footbridges located on federal highway BR-101/PE, by applying the concept of service life established by ISO 15686 (2011), NBR 15575-1 (ABNT, 2021) e NBR 15575-2 (ABNT, 2013). For this purpose, data obtained from the DNIT's Special Structures Management System (SGO) were used. The
\end{abstract}


information on the anomalies and the respective structural stability conditions enabled the use of the Factor Method to obtain the estimated and residual service life of the studied works. The results obtained showed that the methodology can be employed in case studies in which there is no history of regular inspections of SES.

Keywords: Pedestrian walkways; Highway; Deterioration; Inspection; Service life.

\section{Resumen}

El modo de transporte por carretera se destaca como el sistema más utilizado en Brasil para el transporte de cargas y personas. Por lo tanto, es extremadamente importante asegurar los recursos para el mantenimiento de las autopistas y sus Estructuras Artísticas Especiales - EAE (puentes, viaductos y pasos elevados para peatones). Estos últimos, que son el objeto de este estudio, desempeñan un papel indispensable para la seguridad y la comodidad de los usuarios que cruzan las carreteras. Sin embargo, es posible comprobar que un número considerable de estas estructuras se encuentran en muy mal estado de conservación, ya sea por la acción del deterioro que se produce con el paso del tiempo, por deficiencias estructurales derivadas del choque de vehículos, por vandalismo, o por otros factores que afectan a su vida útil. En este sentido, este trabajo tiene como objetivo estimar la vida útil de dos pasarelas de hormigón situadas en la carretera federal BR-101/PE, aplicando el concepto de vida útil establecido por las normas ISO 15686 (2011), NBR 15575-1 (ABNT, 2021) y NBR 15575-2 (ABNT, 2013). Para ello, se utilizaron los datos obtenidos del Sistema de Gestión de Obras de Arte Especiales (SGO) del DNIT. La información de las anomalías y las respectivas condiciones de estabilidad estructural permitieron utilizar el Método de los Factores para obtener la vida útil estimada y residual de las obras estudiadas. Los resultados obtenidos mostraron que la metodología puede emplearse en estudios de casos en los que no hay antecedentes de inspecciones periódicas de EAE.

Palabras clave: Puentes peatonales; Carretera; Deterioro; Inspección; Vida útil.

\section{Introdução}

Segundo estudo de tráfego contido no relatório de projeto do DER/PE (2018), o segmento da BR-101/PE (km 51,60 ao km 82,30), também denominado de Contorno do Recife, recebe diariamente uma quantidade média de 67.236 veículos por dia. Devido ao aumento das atuais sobrecargas transmitidas, muitas vezes não previstas em projeto, ao incremento do porte dos veículos e à intensidade do tráfego, verifica-se uma elevada deterioração dessa rodovia e das suas OAEs.

Considerando que o tráfego acentuado de veículos impõe a necessidade de implantação de passarelas para a transposição da rodovia com segurança pelos pedestres nos segmentos urbanos, tais obras também requerem boas condições de conservação que permitam desempenhos estruturais e funcionais adequados, objetivando a integridade dos usuários e dos ocupantes dos veículos que sob elas transitam.

Uma vez que a maioria dos problemas patológicos é oriundo do uso incorreto e da falta de manutenção adequada (Da Silva et al., 2021), a presença dessas anomalias devem ser evitadas. Dessa forma, é importante garantir adequadas condições de estabilidade e funcionalidade a essas estruturas.

Para tanto, além da realização de inspeções periódicas, as quais possibilitam a verificação da situação atual da estrutura (Soares et al., 2021), existem alguns instrumentos de análise que também podem ser empregados para auxiliar a tomada de decisão quanto às intervenções necessárias (reabilitação, recuperação, reforço, dentre outras).

Tais instrumentos, que devem fazer parte de um sistema mais amplo de gestão, estão relacionados aos conceitos de durabilidade, de confiabilidade estrutural e ao ciclo de vida para a estimativa de vida útil das OAEs. Manafpour et al. (2018) afirmam que tais ações podem maximizar a vida útil e diminuir os custos aplicados ao longo do ciclo de vida dessas estruturas, contribuindo assim para o incremento ou desempenho mínimo exigido das rodovias (Karimzadeh \& Shoghli, 2020).

É nesse contexto que este artigo tem como objetivo apresentar os resultados obtidos da aplicação da metodologia das normas ISO 15686 (2011), NBR 15575-1 (ABNT, 2021) e 15575-2 (ABNT, 2013) para determinar a vida útil estimada e residual de duas passarelas de concreto armado localizadas na rodovia BR-101/PE, visto que essas estruturas estão inseridas em pontos 
críticos da malha rodoviária da BR-101/PE e foram avaliadas com notas técnicas que demonstram condições de estabilidade sofrível e ruim.

Com os resultados obtidos nessa pesquisa, destaca-se a necessidade da realização de uma gestão mais eficaz, especialmente a esses tipos de estruturas, considerando as suas características peculiares, por meio da realização de inspeções regulares e manutenções preventivas.

Essas medidas poderão ainda prolongar a durabilidade e a vida útil, também promover uma economia significativa, pela otimização dos recursos geralmente gastos com dispendiosas intervenções. O método utilizado neste estudo faz parte de uma pesquisa mais abrangente em andamento sobre a vida útil residual das pontes e viadutos e passarelas de pedestres das rodovias brasileiras.

\section{Metodologia}

A estimativa de vida útil das duas passarelas de pedestres localizadas na BR-101/PE foi executada por meio da aplicação do conceito de vida útil preconizado na norma ISO 15686 (2011), fez-se uso das informações obtidas através das vistorias técnicas realizadas previamente em 2017 nas OAEs utilizando-se as diretrizes da norma DNIT 010-PRO (2004).

Os dados relativos à incidência das manifestações patológicas e às deficiências estruturais existentes nas duas passarelas analisadas foram obtidos do Sistema de Gerenciamento de Obras de Arte Especiais do DNIT (SGO), assim como do estudo prévio (Silva et al., 2018) que versa sobre a análise de degradação das passarelas consideradas neste artigo.

\subsection{Normas utilizadas para determinação da vida útil das OAEs estudadas}

A estimativa da vida útil foi feita de acordo com a ISO 15686 (2011), NBR 15575-1 (ABNT, 2021) e NBR 15575-2 (ABNT, 2013), cujo breve histórico está descrito a seguir:

Em 1989, o Architectural Institute of Japan - AIJ propôs um guia contendo orientações que auxiliassem a estimativa de vida útil das construções e de seus elementos (Emídio et al., 2014), desenvolvendo assim o Método dos Fatores (Silva, de Brito, \& Gaspar, 2012). O método permite obter a vida útil estimada (VUE), foi normatizado pela ISO 15686 (2011) e é considerado o método mais aplicado para estimativa da vida útil das construções (Silva, de Brito \& Gaspar, 2016b).

Para o cálculo da VUE, deve-se, multiplicar a Vida Útil de Referência (VUR) por sete fatores que representam os atributos que influenciam na durabilidade da estrutura ou de seus componentes (Loli, Bertolin \& Kotova, 2020; Fregonara \& Pattono, 2018; Souza et al., 2018; Serralheiro, de Brito \& Silva, 2017; Silva, de Brito \& Gaspar, 2016a; Galbusera, de Brito \& Silva, 2015). A VUE pode ser calculada conforme a Equação 1:

$$
V U E=\operatorname{VUR} \times A \times B \times C \times D \times E \times F
$$

Onde:

- $\quad$ VUR - Vida útil de referência

- $\quad$ VUE - Vida útil estimada

- Fator A - Qualidade dos materiais empregados na construção

- $\quad$ Fator B - Nível de qualidade do projeto

- $\quad$ Fator C - Nível de qualidade da execução

- Fator D - Características do ambiente interno

- Fator E-Características do ambiente externo 
- Fator F - Condições de uso

- $\quad$ Fator G - Nível de manutenção

No que tange à VUR, Serralheiro, de Brito \& Silva (2017) destacam que pode ser considerada como a vida útil esperada em condições normais de uso. Janjua, Sarker \& Biswas (2019), Matos (2007) e Zarzar Júnior (2007) mencionam que a VUR propicia subsídios para a estimativa da vida útil de uma construção ou de seus componentes. Segundo esses mesmos autores, alguns aspectos deverão ser levados em consideração nessa análise: a) os agentes de degradação; b) os efeitos da intensidade e da variação da ação desses agentes; c) a sobreposição dos efeitos da ação de vários deles.

Existem variáveis que também estão contempladas na norma ISO 15686 (2011) em três níveis e estão intimamente ligadas ao nível de predomínio exercido sobre o mesmo fator. Para cada uma dessas variáveis pode ser atribuído um peso conforme o que segue:

- 0,80 - Para situações muito desfavoráveis à estrutura ou ao componente estudado;

- 1,00 - Para atributos nas condições que foram suspostas para a vida útil ser igual à VUR;

- 1,20 - Para situações muito favoráveis à estrutura ou ao componente estudado.

A quantificação dos fatores de durabilidade é feita através da imputação de valores extremos $(0,80$ e 1,20$)$ relacionados à VUR. Entretanto, valores intermediários podem ser utilizados de acordo com as condições da estrutura. Silva \& Prieto (2021), Souza et al. (2018) e Silva, de Brito \& Gaspar (2016c) aplicaram valores intermediários de ponderação em suas pesquisas.

\subsection{Coleta das informações sobre as OAEs estudadas}

A Norma DNIT 010-PRO (2004) padroniza as condições para a realização de inspeções em pontes, viadutos, pontilhões e bueiros de concreto estrutural (armado e protendido), assim como a apresentação dos resultados obtidos. Nesse sentido, em decorrência de inspeções visuais realizadas, esse normativo prevê a atribuição de uma nota técnica de avaliação à OAE, variando de 1 a 5, sendo 1 para a pior condição de avaliação e 5 para estruturas sem problemas.

Dessa forma, a gravidade dos problemas identificados na vistoria (anomalias, danos e insuficiências estruturais) influencia diretamente na gradação da nota imputada à OAE analisada.

Tabela 1. Notas técnicas atribuídas às OAEs analisadas.

\begin{tabular}{cccc}
\hline $\begin{array}{c}\text { Estado } \\
\text { federativo }\end{array}$ & Rodovia & Estrutura & Nota Técnica \\
\hline \multirow{2}{*}{ PE } & 101 & Passarela de pedestres km 69,2 & 2 \\
\cline { 3 - 4 } & & Passarela de pedestres km 76,9 & 1 \\
\hline
\end{tabular}

Fonte: Autores (2021).

Sendo assim, a Tabela 1 apresenta as notas técnicas atribuídas às passarelas de pedestres objeto deste estudo, decorrentes de vistorias realizadas pelo DNIT no ano de 2017. As estruturas foram classificadas com notas técnicas 1 (Precária - quando há risco tangível de colapso estrutural) e 2 (Sofrível - quando há significativo dano estrutural, porém sem apresentar risco tangível de colapso).

Portanto, estimar a vida útil de OAEs classificadas com tais notas técnicas é uma ferramenta importante para a tomada de decisão quanto à escolha das intervenções para sua recuperação e adequação estrutural. 


\subsection{Objeto de estudo}

A seguir, são feitas as descrições sumárias das geometrias e sistemas estruturais das OAEs analisadas neste trabalho.

\subsubsection{Passarela de pedestres localizada no $\mathrm{km}$ 69,20}

Trata-se de estrutura construída em concreto armado com 259,13 m de comprimento e largura de 2,32 m. É constituída por um vão de transposição de 45,90 m, o qual está apoiado em quatro pilares com altura máxima de 6,56 m, e por quatro rampas de acesso, sendo duas para cada lado da rodovia em sentidos opostos.

Figura 1. Vista geral da passarela.

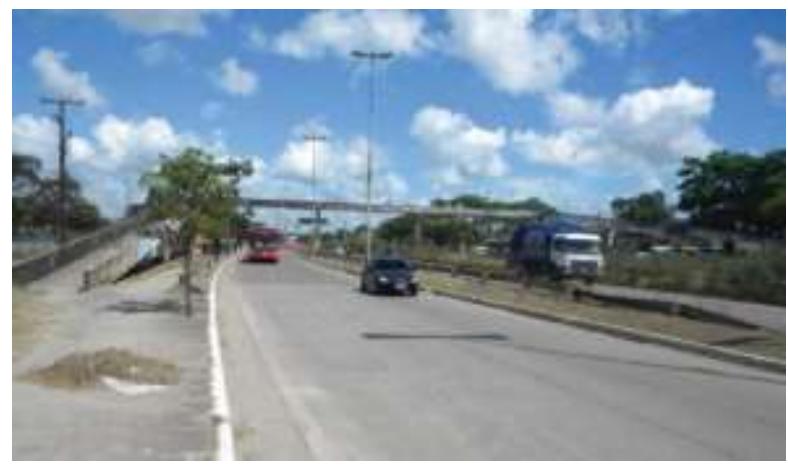

Fonte: SGO (DNIT/2017).

Figura 2. Vista lateral do trecho em rampa da passarela.

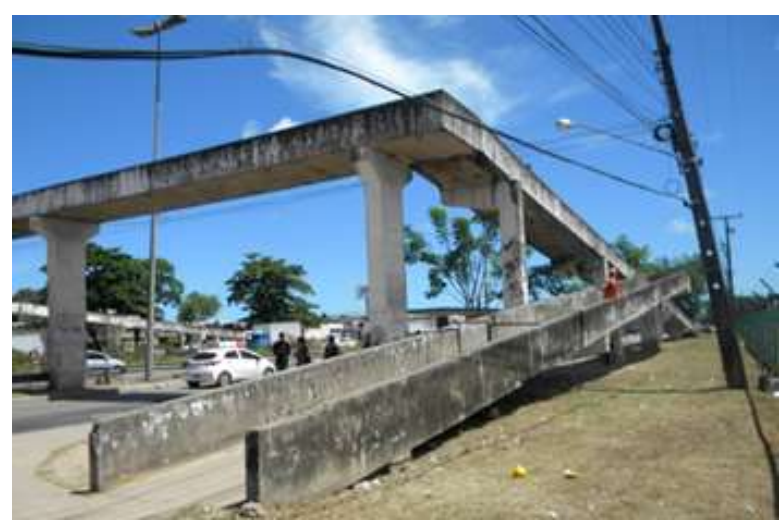

Fonte: SGO (DNIT/2017)

A Figura 1 mostra a vista geral da estrutura estudada e a Figura 2 exibe a vista lateral da passarela a qual destaca os trechos em rampa.

\subsubsection{Passarela de pedestres localizada no $\mathrm{km} 76,90$}

Trata-se de uma passarela executada em estrutura de concreto armado com 175,70 m de comprimento e largura de 1,90 m. É constituída por três vãos, dentre os quais as rampas de acesso. O vão de transposição da rodovia possui $22,00 \mathrm{~m}$ de extensão e a altura máxima dos dois pilares centrais de concreto que o apoiam é de 7,00 m. 
Figura 3. Vista geral da passarela.

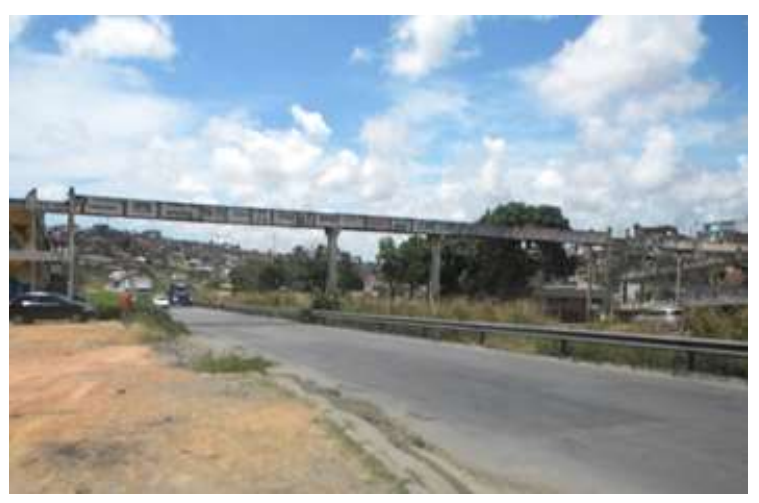

Fonte: SGO (DNIT/2017).

Figura 4. Vista do trecho em rampa da passarela.

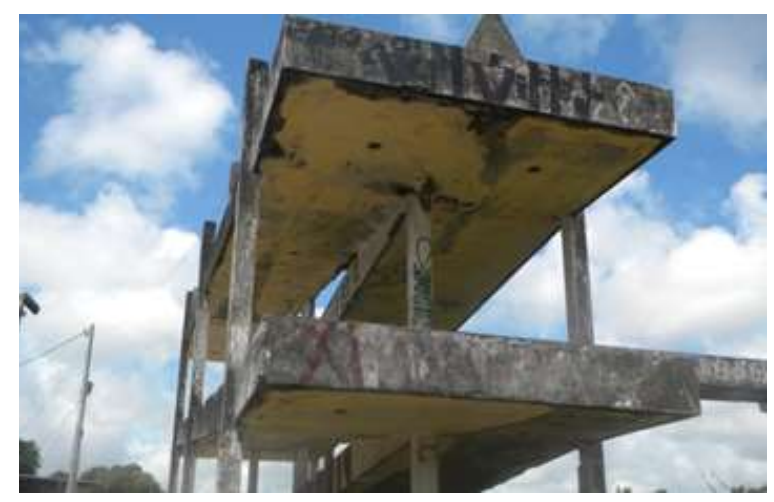

Fonte: SGO (DNIT/2017)

As Figuras 3 e 4 apresentam respectivamente a vista geral da passarela, com destaque para o trecho de transposição da rodovia, e a vista de um dos trechos em rampa.

\section{Resultados e Discussões}

\subsection{Manifestações patológicas e deficiências estruturais observadas nas passarelas estudadas}

São apresentadas a seguir as manifestações patológicas e os danos estruturais identificados que subsidiaram a estimativa de vida útil das passarelas de pedestres estudadas.

\subsubsection{Passarela de pedestres do $\mathrm{km} \mathrm{69,20}$}

As principais manifestações patológicas e deficiências observadas na estrutura estão destacadas conforme as Figuras 5 a 8. 
Figura 5. Face lateral da viga invertida exibindo um trecho contendo deterioração do concreto e exposição de armaduras em elevado grau de corrosão.

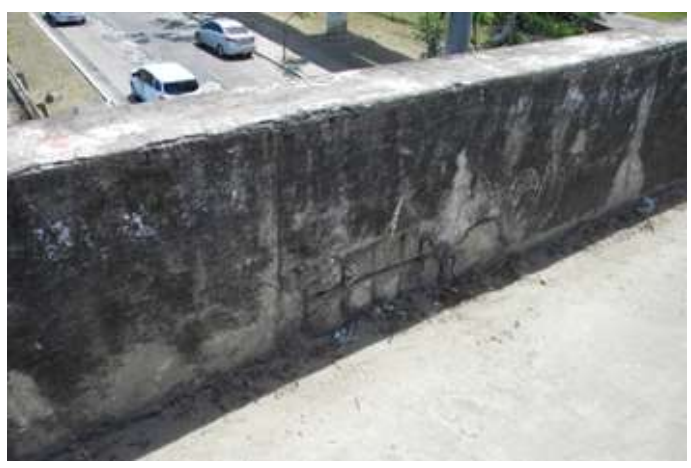

Fonte: SGO (DNIT/2017)
Figura 6. Degradação do concreto dos pilares com elevada exposição e corrosão de armaduras. Verificam-se perdas de seção e ruptura dos ferros de flexão e cisalhamento em um trecho do pilar.

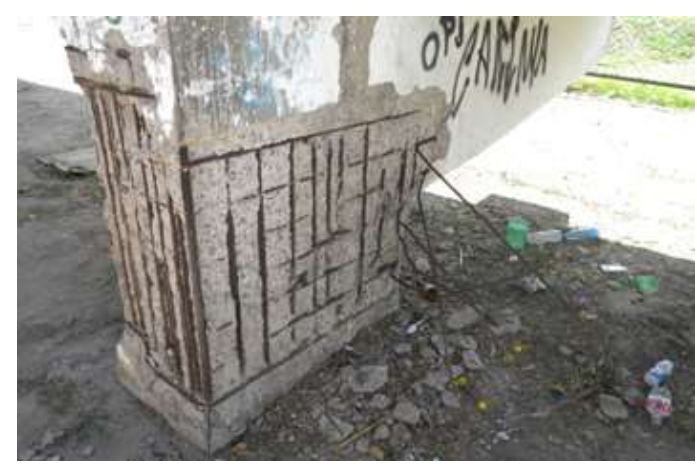

Fonte: SGO (DNIT/2017).

As Figuras 5 e 6 exibem a viga invertida, que funciona como guarda-corpo para passarela estudada, e a parte inferior de um dos pilares da estrutura apresentando degradação do concreto e elevado grau de corrosão de armaduras, evidenciando ainda uma acentuada redução de seção e ruptura das armaduras de flexão e de cisalhamento do pilar.

Figura 7. Extremidades das vigas da passarela apresentando armaduras totalmente expostas.

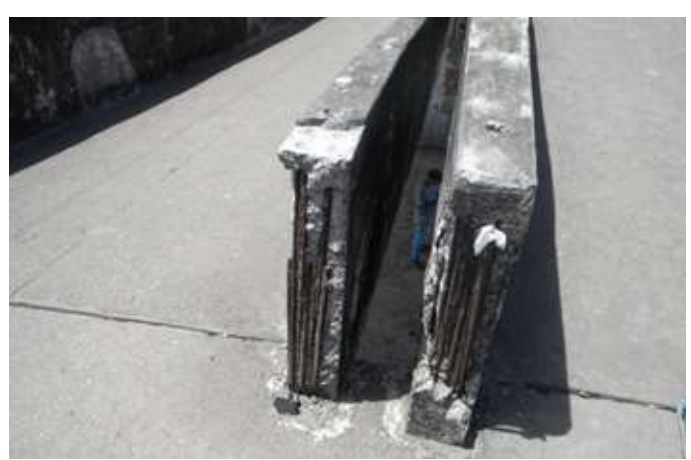

Fonte: SGO (DNIT/2017).
Figura 8. Deterioração do concreto com exposição de armaduras na face inferior das vigas do tabuleiro.

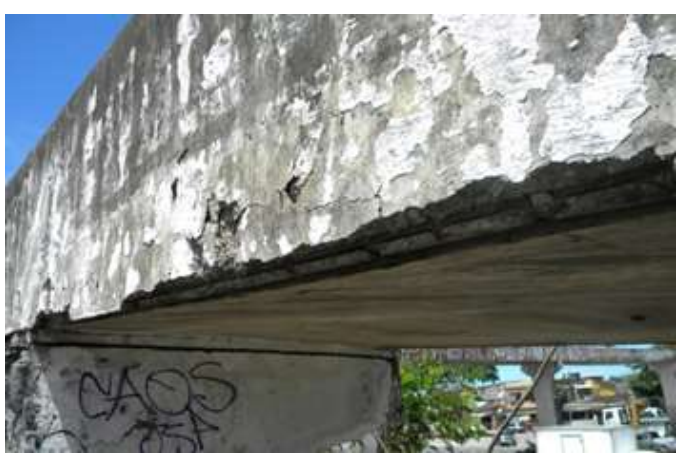

Fonte: SGO (DNIT/2017)

Além da deterioração identificada na extensão da viga invertida (Figura 5), também foram verificadas armaduras totalmente expostas nas extremidades das vigas da passarela (Figura 7). Há ainda degradação do concreto com exposição de armaduras na face inferior das vigas de tabuleiro conforme ilustrado na Figura 8.

\subsubsection{Passarela de pedestres $\mathrm{do} \mathrm{km} 76,90$}

As principais anomalias e problemas estruturais observados nessa estrutura estão contemplados nas Figuras 9 a 12. 
Figura 9. Detalhe da região do dente Gerber apresentando deslocamento lateral entre as estruturas do tabuleiro.

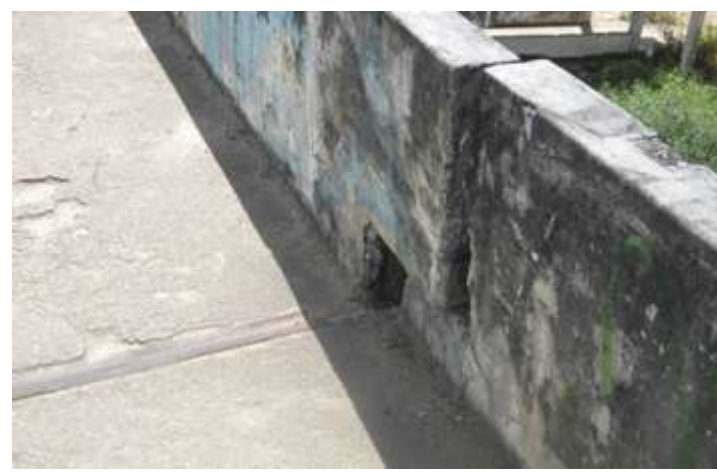

Fonte: SGO (DNIT/2017)
Figura 10. Ligação da laje do passeio com o pilar apresentando danos no concreto e perda de seção de armaduras transversais.

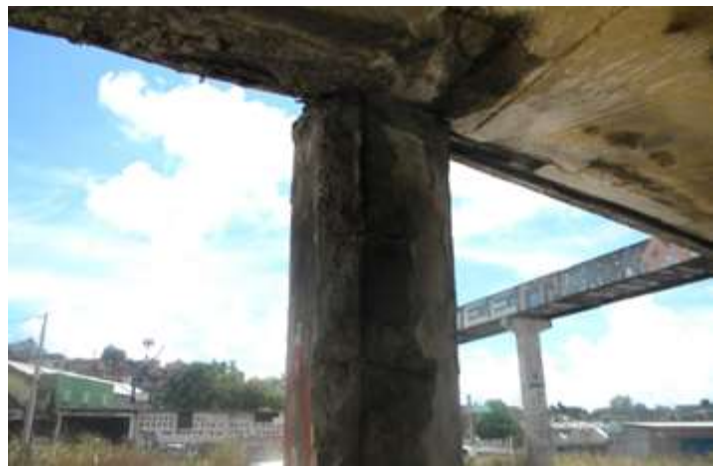

Fonte: SGO (DNIT/2017).

A Figura 9 destaca a região de dente Gerber na qual houve um deslocamento lateral entre as estruturas do tabuleiro provavelmente ocasionado pelo impacto de veículo que transita pela rodovia. Observaram-se na ligação da laje do passeio com o pilar danos no coreto e perda de seção de armaduras transversais decorrentes da deficiência do sistema de drenagem do tabuleiro (Figura 10).

Figura 11. Detalhe da junta sobre dente Gerber com abertura excessiva na região do apoio, onde o concreto encontra-se deteriorado.

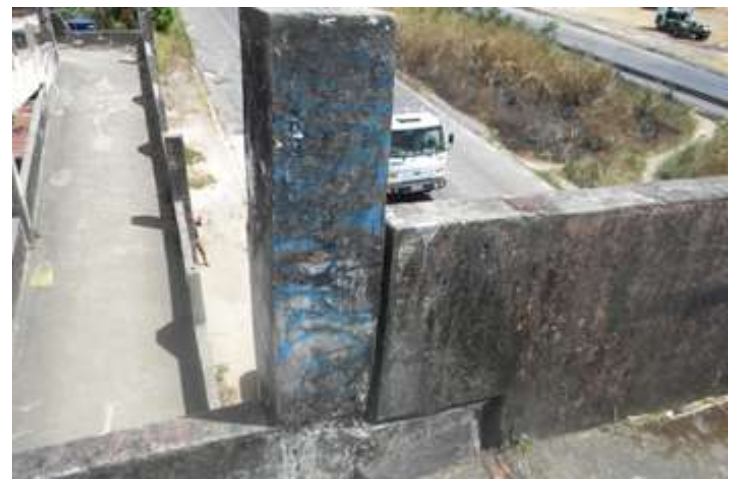

Fonte: SGO (DNIT/2017)
Figura 12. Manchas de umidade e eflorescências na face inferior do tabuleiro oriundos da deficiência do sistema de drenagem do tabuleiro.

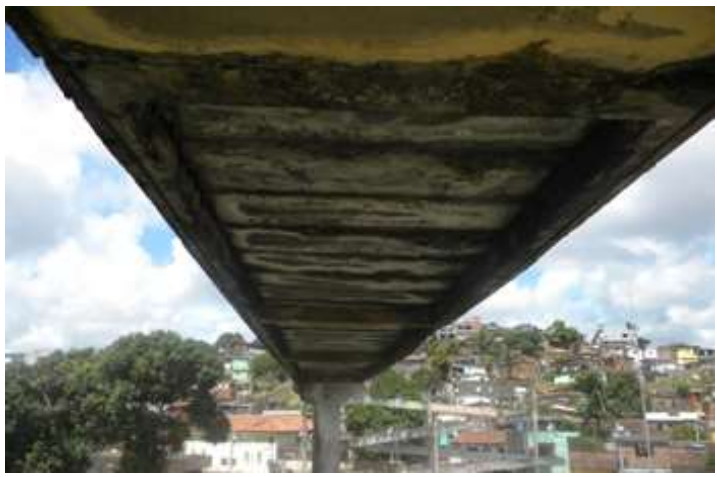

Fonte: SGO (DNIT/2017).

Verificou-se também abertura excessiva na região de um dos apoios, onde o concreto se encontra deteriorado (Figura 11). Foram constatadas manchas de umidade e eforescências na face inferior do tabuleiro decorrentes da deficiência do sitema de drenagem do tabuleiro (Figura 12). 


\subsection{Estimativa de vida útil residual das OAES analisadas}

A partir das manifestações patológicas e deficiências observadas nas passarelas do presente estudo e evidenciadas nas Figuras 5 a 12, aplicou-se o conceito de vida útil definido na norma ISO 15686 (2011) e preconizado pelas normas ABNT NBR 15575-1 (2021) e 15575-2 (2013).

Para aplicação do Método dos Fatores às passarelas analisadas, adotou-se como parâmetro a Vida Útil de Projeto (VUP) mínima de 50 anos prevista na Tabela C.6 - Exemplos de vida útil de projeto (VUP) da ABNT NBR 15.575-1 (2021), tendo em vista que ainda não existe no Brasil norma ou outra literatura técnica que especifique tal período de vida para pontes, viadutos e passarelas de pedestres.

Em seguida, foi realizada uma adaptação dos fatores às condições de exposição das passarelas.

Tabela 2. Critérios adotados para aplicação do Método dos Fatores.

\begin{tabular}{|c|c|}
\hline Fator & Critérios considerados \\
\hline $\mathbf{A}$ & $\begin{array}{l}\text { - Qualidade e aparência do concreto utilizado para proteção } \\
\text { contra intempéries no que concerne ao seu desempenho }\end{array}$ \\
\hline B & $\begin{array}{l}\text { - O projeto foi executado de acordo com os normativos } \\
\text { vigentes à época da sua elaboração (dimensionamento, } \\
\text { cobrimento, materiais adequados à solução, gabarito } \\
\text { vertical, drenagem, dentre outros) }\end{array}$ \\
\hline $\mathbf{C}$ & $\begin{array}{l}\text { - Análise do método de execução da estrutura } \\
\text { - O método de execução permite o registro de manutenções }\end{array}$ \\
\hline D & $\begin{array}{l}\text { - Por se tratar de uma estrutura localizada num meio externo, } \\
\text { não foram considerados aspectos atinentes a este fator }\end{array}$ \\
\hline $\mathbf{E}$ & $\begin{array}{l}\text { - Análise dos aspectos relacionados à degradação da estrutura } \\
\text { (Presença de umidade, eflorescências, bolor, condições de } \\
\text { agressividade ambiental, existência de agentes agressivos, } \\
\text { tais como, cloretos, sulfatos, CO2, localização em ambientes } \\
\text { de grandes variações de temperatura, presença de erosões) }\end{array}$ \\
\hline $\mathbf{F}$ & $\begin{array}{l}\text { - Verificação da estrutura quanto à sua funcionalidade } \\
\text { - Grandes variações de cargas } \\
\text { - Nível de vibração do tabuleiro (normal, intenso e/ou } \\
\text { - } \quad \text { Exagerado) } \\
\text { - Ustência de vandalismo } \\
\text { - Usorreto (moradia irregular sob a estrutura) }\end{array}$ \\
\hline $\mathbf{G}$ & $\begin{array}{l}\text { - Realização de manutenções preventivas } \\
\text { - Registro de manutenções } \\
\text { - Implementação de programa de manutenção } \\
\text { - Realização de manutenções corretivas } \\
\text { - Existência de contrato de manutenção }\end{array}$ \\
\hline
\end{tabular}

Tomando-se como base os critérios considerados para cada fator na Tabela 2 e a observação das manifestações patológicas e dos danos estruturais, foram imputados os pesos das variáveis de cada fator aplicados às passarelas da presente pesquisa.

Em relação à passarela do $\mathrm{km} \mathrm{69,20} \mathrm{foi} \mathrm{imputado} \mathrm{o} \mathrm{valor} \mathrm{0,90} \mathrm{para} \mathrm{os} \mathrm{Fatores} \mathrm{A,} \mathrm{B,} \mathrm{E} \mathrm{e} \mathrm{F,} \mathrm{visto} \mathrm{que} \mathrm{essa} \mathrm{estrutura}$ apresentou deficiências relacionadas à qualidade dos materiais, a falhas de projeto (ausência de buzinotes os quais geraram 
infiltrações e eflorescências), ao ambiente externo (alta agressividade ambiental) e a problemas concernentes às condições de uso. No tocante ao Fator D, foi indicado um valor 1,00 por entender que tal ambiente não interfere na análise. O valor da variável para o Fator $\mathrm{G}$ foi indicado como 0,80 , visto que há diversos aspectos referentes à sua degradação e à sua manutenção que contribuem negativamente para a estrutura estudada. O Fator $\mathrm{C}$ foi qualificado com peso 1,10.

No que tange à passarela do km 76,90, foram atribuídos os pesos 1,10 (Fator C), 1,00 (Fator D), 0,90 (Fatores A e E) e o valor 0,80 para os Fatores B, F e G respeitando-se os mesmos critérios mencionados anteriormente na análise da passarela do $\mathrm{km} \mathrm{69,20.}$

Tabela 3. Peso das variáveis aplicadas por fator para cada passarela estudada.

\begin{tabular}{cccc}
\hline \multicolumn{2}{c}{ Passarela de pedestres km 69,20 } & \multicolumn{2}{c}{ Passarela de pedestres km 79,60 } \\
\hline Fator & $\begin{array}{r}\text { Peso da } \\
\text { variável }\end{array}$ & Fator & $\begin{array}{c}\text { Peso da } \\
\text { variável }\end{array}$ \\
\hline A & 0,90 & A & 0,90 \\
B & 0,90 & B & 0,80 \\
C & 1,10 & C & 1,10 \\
D & 1,00 & D & 1,00 \\
E & 0,90 & E & 0,90 \\
F & 0,90 & F & 0,80 \\
G & 0,80 & G & 0,80 \\
\hline
\end{tabular}

Fonte: Autores (2021).

A Tabela 3 abrange o peso atribuído às variáveis de cada fator das duas passarelas estudadas. Ressalta-se que não foi imputado o valor 1,20 para nenhum dos sete fatores, visto que as OAEs analisadas não apresentaram condições muito favoráveis à estrutura ou ao componente estudado.

\subsection{Determinação da vida útil estimada das OAEs estudadas}

\subsubsection{Cálculo da vida útil estimada}

Os resultados oriundos da atribuição dos pesos das variáveis para cada fator das duas passarelas mostrados na Tabela 3 foram multiplicados pela vida útil de referência para obtenção da VUE. A Tabela 4 apresenta uma síntese dos valores da VUE das duas estruturas para 50, 63 e 75 anos de VUR.

Tabela 4. Síntese dos valores da VUE das passrelas do presente estudo

\begin{tabular}{ccc}
\hline Estrutura & $\begin{array}{c}\text { VUR } \\
\text { (Anos) }\end{array}$ & $\begin{array}{c}\text { VUE } \\
\text { (Anos) }\end{array}$ \\
\hline \multirow{2}{*}{$\begin{array}{c}\text { Passarela de pedestres } \\
\text { km 69,2 }\end{array}$} & 50 & 28,87 \\
\cline { 2 - 3 } & 63 & 36,37 \\
\cline { 2 - 3 } & 75 & 43,30 \\
\hline Passarela de pedestres & 50 & 22,81 \\
\cline { 2 - 3 } km 79,6 & 63 & 28,74 \\
\cline { 2 - 3 } & 75 & 34,21 \\
\hline
\end{tabular}

Fonte: Autores (2021). 
Observa-se na Tabela 4 que a passarela de pedestres - km 69,20 apresentou vida útil estimada de 28,87, 36,37 e 43,30 anos, as quais foram calculadas para VUR de 50, 63 e 75 anos, respectivamente. A outra passarela exibiu valores bem mais dispersos da vida útil de referência adotada (22,81, 28,74 e 34,21 anos), quando calculadas para os mesmos períodos de VUR.

\subsubsection{Cálculo da vida útil residual}

Uma vez que a BR-101/PE foi executada em meados dos anos 40 e as OAEs do trecho estudado foram construídas no final dos anos 70, considerou-se 1980 como o ano de construção das estruturas analisadas conforme informações aproximadas obtidas junto ao corpo de engenheiros do DER/PE. Dessa forma, a Tabela 5 exibe uma síntese da vida útil residual das passarelas estudadas para 50, 63 e 75 anos com as respectivas reduções de VUR.

Tabela 5. Síntese da vida útil residual das passarelas analisadas.

\begin{tabular}{cccccccc}
\hline Estrutura & $\begin{array}{c}\text { VUR } \\
\text { (Anos) }\end{array}$ & $\begin{array}{c}\text { VUE } \\
\text { (Anos) }\end{array}$ & $\begin{array}{c}\text { Redução } \\
\text { da VUR } \\
(\%)\end{array}$ & $\begin{array}{c}\text { Ano de } \\
\text { construção }\end{array}$ & $\begin{array}{c}\text { Vida útil } \\
\text { residual }\end{array}$ & $\begin{array}{c}\text { Situação } \\
\text { atual }\end{array}$ & $\begin{array}{c}\text { Decisão a } \\
\text { adotar }\end{array}$ \\
\cline { 2 - 7 } $\begin{array}{c}\text { Passarela de pedestres } \\
\text { km 69,20 }\end{array}$ & 50 & 28,87 & 42,26 & 1980 & 2009 & $\begin{array}{c}\text { Período de } \\
\text { sobrevida }\end{array}$ & $\begin{array}{c}\text { Intervenção } \\
\text { imediata }\end{array}$ \\
\cline { 2 - 7 } & 75 & 36,37 & 42,26 & 1980 & 2016 & $\begin{array}{c}\text { Período de } \\
\text { sobrevida }\end{array}$ & $\begin{array}{c}\text { Intervenção } \\
\text { imediata }\end{array}$ \\
\hline $\begin{array}{c}\text { Passarela de pedestres } \\
\text { km 79,60 }\end{array}$ & 50 & 22,81 & 54,38 & 1980 & 2003 & $\begin{array}{c}\text { Período de } \\
\text { sobrevida }\end{array}$ & $\begin{array}{c}\text { Intevenção } \\
\text { imediata }\end{array}$ \\
\hline & 63 & 28,74 & 54,38 & 1980 & 2009 & $\begin{array}{c}\text { Período de } \\
\text { sobrevida }\end{array}$ & $\begin{array}{c}\text { Intervenção } \\
\text { imediata }\end{array}$ \\
\hline
\end{tabular}

Fonte: Autores (2021).

A vida útil estimada de cada passarela foi calculada através da multiplicação do conjunto dos pesos dos sete fatores pela vida útil de referência aos 50, 63 e 75 anos. Após essa operação, foi obtida a vida útil residual para os períodos de VUR anteriormente mencionados, adotando-se como referência o ano de construção (1980). Verificou-se ainda que houve uma diminuição considerável da VUR de ambas as passarelas de pedestres (42,26\% e 54,38\%). Esses dados estão exibidos na Tabela 5. As discussões relativas à situação atual e à decisão a adotar dessas estruturas estão contidas nas considerações finais deste estudo.

\section{Considerações Finais}

Neste trabalho foi feito um estudo sobre a vida útil residual de duas passarelas de pedestres localizadas na BR-101/PE, por meio da aplicação do conceito de vida útil, tendo como base as informações dos relatórios técnicos de inspeção e registros fotográficos obtidos diretamente do banco de dados do DNIT e da análise prévia sobre a degradação dessas estruturas realizada por Silva et al. (2018).

Mesmo considerando as limitações do estudo realizado, é possível concluir que a passarela de pedestres do km 69,20 apresenta problemas patológicos generalizados, associados à falta de manutenção, cujas implicações indicam uma deficiência estrutural expressiva que pode afetar tanto a segurança da obra como a redução do restante da sua vida útil. Esse fato pode ser 
confirmado pelo simples estado de conservação de alguns pilares que apresentam um elevado nível de corrosão de armaduras, com perda de aderência e de seção transversal.

No tocante à passarela do km 76,90, além da identificação dos mesmos problemas da obra do km 69,20 verificaram-se danos que podem provocar insuficiência estrutural, caso não sejam adotadas medidas urgentes para eliminá-los, considerando que isso poderá afetar inevitavelmente a sua vida útil. Um exemplo concreto é o grande deslocamento lateral com evidências de rotação dos apoios em dentes Gerber, possivelmente gerado por impacto de veículos.

Quanto à análise da vida útil estimada, foram adotados os períodos de 50, 63, e 75 anos de vida útil de referência previstos na NBR 15575-1 (2021) para determinar a vida útil residual das estruturas analisadas. Considerando os resultados obtidos é possível fazer as seguintes conclusões:

- A passarela de pedestres do km 69,20 encontra-se no período de sobrevida ao se adotarem 50 e 63 anos como VUR, tendo atingido sua vida útil residual em 2009 e 2016, respectivamente. Em contrapartida, ao se considerar como 75 anos de VUR, a estrutura alcançará a sua vida útil residual em 2023.

- A passarela de pedestres do $\mathrm{km} \mathrm{76,90} \mathrm{requer} \mathrm{intervenção} \mathrm{imediata,} \mathrm{pelo} \mathrm{fato} \mathrm{de} \mathrm{se} \mathrm{encontrar} \mathrm{no} \mathrm{período} \mathrm{de} \mathrm{sobrevida}$ ao serem adotados os períodos de 50,63, e 75 anos de vida útil de referência (Redução de 54,38\%).

Embora as metodologias utilizadas nesta pesquisa apresentem características peculiares na exibição dos resultados, ambas consideram os danos e fatores que afetam a durabilidade no momento da análise das estruturas estudadas. Entretanto, é possível concluir que a aplicação do Método dos Fatores permitiu uma estimativa mais realista das condições de estabilidade e conservação das passarelas estudadas, e que pode ser estendida às demais OAEs da malha viária brasileira, como as pontes e os viadutos, por se tratar de uma metodologia com maior variabilidade dos parâmetros utilizados, o que proporciona a obtenção da vida útil estimada e também a verificação da redução da vida útil de referência e, por conseguinte, da vida útil residual de cada estrutura.

No que tange às OAEs do presente estudo, ambas tecnicamente podem ser recuperadas sem necessidade de demolição através de técnicas de reforço estrutural da meso e superestrutura, incluindo a reabilitação dos dentes Gerber e cobrimento do concreto. A intervenção nas passarelas estudadas também deverá considerar um possível reforço na fundação, além da adequação da altura dos guarda-corpos, a instalação de alambrado lateral, de cobertura metálica e de dispositivos de iluminação para permitir a travessia noturna aos usuários.

Como sugestão para trabalhos futuros, recomenda-se aplicar o método dos fatores a outras tipologias construtivas (pontes e viadutos), realizar uma análise comparativa entre o uso desse método com modelos probabilísticos que também permitam estimar a sua vida útil. Para estruturas que contenham um alto grau de degradação, aconselha-se ainda avaliar a viabilidade técnica e econômica das estruturas no tocante à sua recuperação perante a demolição, verificando, inclusive, os transtornos que poderão ser causados aos transeuntes.

Destaca-se ainda que a metodologia empregada nesta pesquisa está inserida em um estudo mais abrangente em desenvolvimento, cujo fito também é permitir uma análise comparativa através da utilização de outros métodos - inclusive os probabilísticos - a fim de avaliar as condições de segurança e determinação da vida útil de pontes, viadutos e passarelas de pedestres existentes.

Por fim, os dados obtidos neste trabalho demonstraram que a metodologia aplicada para análise da vida útil pode ser usada especialmente em situações onde não se dispõe de registros de inspeções regulares, contribuindo assim para a tomada de decisão no que se refere à otimização de recursos e ao planejamento futuro de manutenções preventivas. 


\section{Agradecimentos}

Os autores agradecem ao Departamento Nacional de Infraestrutura de Transportes (DNIT) por disponibilizar as informações necessárias à execução dessa pesquisa.

\section{Referências}

Associação Brasileira de Normas Técnicas. NBR 15575. (2021). Edificações Habitacionais - Desempenho Parte 1: Requisitos Gerais. Rio de Janeiro, Brasil.

Associação Brasileira de Normas Técnicas. NBR 15575. (2013). Edificações Habitacionais - Desempenho Parte 2: Requisitos para os sistemas estruturais. Rio de Janeiro, Brasil.

Da Silva, H. T., Guimarães, L. S., Júnior, D. S. T., Campos, A. S., Frisso, A. L., da Costa, A. S. V., Cabral, S. C., \& Freitas, L. F. (2021). Restoration proposal for correction of pathologies in concrete bridge-case study in the city of Teófilo Otoni, Brazil. Research, Society and Development, 10(6), e21810615767e21810615767. DOI: $10.33448 /$ rsd-v10i6.15767.

Departamento de Estradas de Rodagem de Pernambuco. (2018). Elaboração do projeto executivo de engenharia e execução das obras e demais operações necessárias e suficientes para as obras de melhoramentos, adequação de capacidade e segurança e reabilitação do pavimento. Relatório técnico. Recife.

Departamento Nacional de Infraestrutura de Transportes. (2004). DNIT 010/2004-PRO: Inspeções em pontes e viadutos de concreto armado e protendido Procedimento.

Departamento Nacional de Infraestrutura de Transportes. (2017). Sistema de gestão de Obras de Arte Especiais - SGO. Inspeção rotineira, dados cadastrais e relatório fotográfico. Brasília.

Emídio, F., de Brito, J., Gaspar, P. L., \& Silva, A. (2014). Application of the factor method to the estimation of the service life of natural stone cladding. Construction and Building Materials, 66, 484-493. DOI: 10.1016/j.conbuildmat.2014.05.073.

Fregonara, E., \& Pattono, S. (2018). A sustainability indicator for building projects in presence of risk/uncertainty over time: a research experience. Aestimum, 173-205. DOI: 10.13128/Aestimum-24928.

Galbusera, M. M., de Brito, J., \& Silva, A. (2015). Application of the factor method to the prediction of the service life of ceramic external wall cladding. Journal of Performance of Constructed Facilities, 29(3), 04014086. DOI: 10.1061/(ASCE)CF.1943-5509.0000588.

International Organization for Standardization. (2011). ISO 15.686-1: Buildings and constructed assets - Service life planning - Part 1: General principles and framework.

Janjua, S., Sarker, P., \& Biswas, W. (2019). A review of residential buildings' sustainability performance using a life cycle assessment approach. Journal of Sustainability Research, 1(1), 1-29. DOI: 10.20900/jsr20190006.

Karimzadeh, A., \& Shoghli, O. (2020). Predictive analytics for roadway maintenance: A review of current models, challenges, and opportunities. Civil Engineering Journal, 6(3), 602-625. DOI: 10.28991/cej-2020-03091495.

Loli, A., Bertolin, C., \& Kotova, L. (2020). Service life prediction of building components in the times of climate change. In IOP Conference Series: Materials Science and Engineering (Vol. 949, No. 1, p. 012048). IOP Publishing. DOI: 10.1088/1757-899X/949/1/012048.

Manafpour, A., Guler, I., Radlińska, A., Rajabipour, F., \& Warn, G. (2018). Stochastic analysis and time-based modeling of concrete bridge deck deterioration. Journal of Bridge Engineering, 23(9), 04018066. DOI: 10.1061/(ASCE)BE.1943-5592.0001285.

Matos, M. J. D. S. (2007). Durabilidade como critério de projecto: o método factorial no contexto português. (Dissertação de mestrado), Departamento de Engenharia Civil, Faculdade de Engenharia da Universidade do Porto. https://repositorio-aberto.up.pt/bitstream/10216/12402/2/Texto\%20integral.pdf.

Serralheiro, M. I., de Brito, J., \& Silva, A. (2017). Methodology for service life prediction of architectural concrete facades. Construction and Building Materials, 133, 261-274. DOI: 10.1016/j.conbuildmat.2016.12.079.

Silva, A., de Brito, J., \& Gaspar, P. L. (2012). Application of the factor method to maintenance decision support for stone cladding. Automation in Construction, 22, 165-174. DOI: 10.1016/j.autcon.2011.06.014.

Silva, A., de Brito, J., \& Gaspar, P. L. (2016a). Stochastic approach to the factor method: durability of rendered façades. Journal of Materials in Civil Engineering, 28(2), 04015130. DOI: 10.1061/(ASCE)MT.1943-5533.0001409.

Silva, A., de Brito, J., \& Gaspar, P. L. (2016b). Comparative analysis of service life prediction methods applied to rendered façades. Materials and Structures, 49(11), 4893-4910. DOI: 10.1617/s11527-016-0832-6.

Silva, A., de Brito, J., \& Gaspar, P. L. (2016c). Deterministic versus probabilistic approach of the factor method for the service life prediction of ceramic claddings. In: Life-Cycle of Engineering Systems: (pp. 1277-1284). CRC Press.

Silva, C. J. G., Vitório, J. A. P., Carneiro, A. M. P., Oliveira, R. A. (2018). Análise da degradação estrutural de duas passarelas de pedestres situadas na BR101/PE. In: $3^{\circ}$ Encontro Luso-Brasileiro de Degradação em Estruturas de Concreto Armado, São Carlos, São Paulo, Brasil. 
Silva, A., \& Prieto, A. J. (2021). Modelling the service life of timber claddings using the factor method. Journal of Building Engineering, 37, 102137. DOI: 10.1016/j.jobe.2020.102137.

Soares, R. G. P., de Oliveira Rodrigues, G. G., De Moraes, I. D. L., Pacheco, C. R. X., de Moura, L. S., do Nascimento, K. M. B., Apolônio, P. H., Carneiro, A. M. P., \& de Oliveira, R. A. (2021). Characterization of the useful life and structural performance of a reinforced concrete bridge in the City of Correntes-PE. Research, Society and Development, 10(5), e4810514579-e4810514579. DOI: 10.33448/rsd-v10i5.14579.

Souza, J., Silva, A., de Brito, J., \& Bauer, E. (2018). Service life prediction of ceramic tiling systems in Brasília-Brazil using the factor method. Construction and building materials, 192, 38-49. DOI: 10.1016/j.conbuildmat.2018.10.084.

Zarzar Júnior, F. C. (2007). Metodologia para estimar a vida útil de elementos construtivos, baseados no método dos fatores. (Dissertação de mestrado), Departamento de Engenharia Civil, Universidade Católica de Pernambuco. http://tede2.unicap.br:8080/handle/tede/26. 